\title{
A Review of Visual Salient Object Detection
}

\author{
XU Hai-shui ${ }^{\mathrm{a}}$, XIA Lin-yue ${ }^{\mathrm{b}}$ \\ School of Computer, Guangdong University of Technology, Guangzhou 510006,China \\ axhs@gdut.edu.cn, b1643207355@qq.com
}

\begin{abstract}
Vision saliency detection has been applied in image preprocessing steps. The purpose of this paper is to introduce the research direction of image saliency detection and introduce the performance evaluation criteria and datasets of the model. Then investigated a variety of methods, introduced various models and classified models including traditional and deep learning methods, the latest developments, and the problems and challenges of saliency models, and summarized and proposed future research direction.
\end{abstract}

Keywords: Salient object detection,image saliency detection,salient model,salient map.

\section{Introduction}

The primate will select a subset of the image for advanced processing before advanced visual processing to reduce the complexity of the scene. This mechanism for selecting parts of the area for attention is called visual salient detection, as show in Fig. 1 .Visual saliency includes both bottom-up and top-down mechanisms. From the bottom up, it can be considered as data-driven, that is, the image itself attracts people. From top to bottom, it is under the control of human consciousness to pay attention to images. Research mainly do from the bottom up visual connotation.Computer vision includes not only saliency detection, image segmentation, object detection, but also the importance and importance of image detection. The salience graph is the result of the saliency detection, and its definition range is the grayscale graph [0.255][1]. Each value represents the prominence of the pixels or regions in the image. The greater the significant value is, the higher the prominence is. On the application of saliency detection, the DSS method [2] is used for HUAWEI mate10's smartphones to take photos.
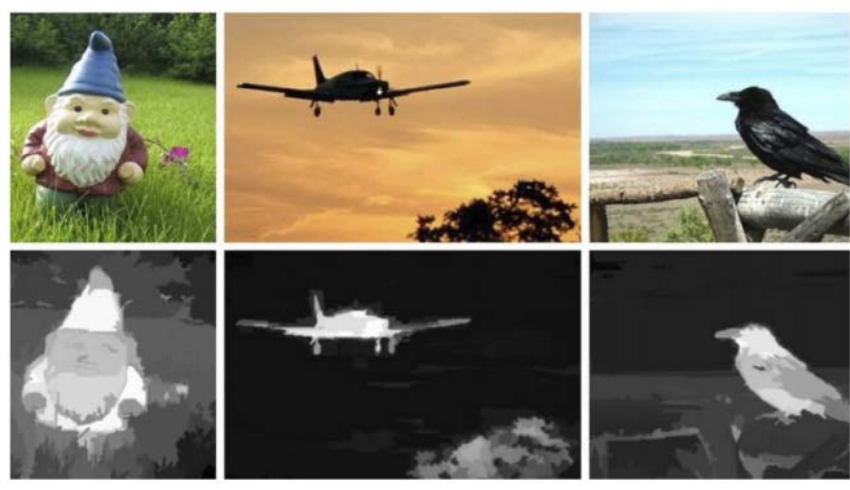

Fig. 1 saliency detection 


\section{Organization of the Training set and evaluation criteria for significant detection}

\subsection{Datasets for saliency detection}

Datasets for saliency detection as show Table 1

Table 1 datasets for saliency detection

\begin{tabular}{|l|l|l|l|}
\hline Dataset of name & Resolving power & Image numbers & year \\
\hline ASD[2] & $300 \times 400,400 \times 300$ & 1000 & 2009 \\
\hline SOD[3] & $300 \times 38$ & 300 & 2010 \\
\hline MSRA10k[4] & $300 \times 400,400 \times 300$ & 10000 & 2011 \\
\hline SOC[5] & $640 \times 480,1024 \times 500$ & 6000 & 2018 \\
\hline
\end{tabular}

\subsection{Evaluation criterion}

After the completion of the significance test, the corresponding evaluation is required.

ROC refers to the curve of the receiver operating characteristic curve, it is a comprehensive index, the sensitivity and specificity of continuous variables, the relationship between sensitivity and specificity revealed by mapping method, it will be set different threshold value through a plurality of continuous variables, which calculated a series of sensitivity and specificity. then, the curve be draw up through sensitivity as the ordinate, (1- specific) as the abscissa, the greater the area under the curve is, the higher diagnostic accuracy is.

AUC is defined as the area under the ROC curve, firstly, the meaning of the AUC value is a probability value, when you randomly pick a positive sample and a negative sample, according to the calculated Score value, AUC is probability that the classification algorithm put the positive samples in the front of negative value. The ROC curve is generally above the line of $y=x$, so the range of AUC is between 0.5 and 1 . The larger the AUC value is, the more the current classification algorithm likely put the positive sample in front of the negative sample is, that is, it can be better classified.

Structure-measure [6] structural similarity index, which is used to measure non-binary foreground mapping. It combines regional and object-oriented structural similarity.

\section{Saliency detection model}

\subsection{Saliency detection model for traditional methods}

To simulate the mechanism of visual attention in biology, Itti et al. [4] obtained the final saliency map by combining multi-scale color, intensity and direction saliency map, which is a significant 
opening work. The traditional method, [5, 6], is used for saliency detection, from significant regional detection based on global contrast (HC and RC). Cheng Mingming's color contrast method uses the color feature of the region and the spatial relation to making a global comparison. Cellular automata based saliency detection [7], where cell is equivalent to every pixel value, and cellular automata can update every cell state through neighbor interactions. Based on the realization of global and local cues, Yang et al. Constructed the top-down saliency model of CRF and [8],and built a complex energy function to achieve it. From the perspective of background and prospect, Jiang and others proposed a saliency detection model based on the absorption Markov chain [9].

In 2007 Tie Liu[10] algorithm using some new features, such as multi-scale contrast, centre around histogram, color spatial distribution characteristics of local, regional, global, and training a CRFs, the algorithm adds some dynamic objects. For the detection of the moving object detection of significant figure. Achanta[11] and the other take advantage of the multiscale significance. Tilke Judd [12] and other algorithms use low level intermediate advanced features for significant detection. Huaizu Jiang [13] and other supervised learning methods are used. Yu Fu [14] and other articles combine the saliency detection and image segmentation and launch an algorithm similar to clippings. Perazzi [15] and other algorithms mainly use the LAB color as a feature. The algorithm proposed by Jiang[16] and mainly used in the UFO algorithm, namely, the uniqueness, the focus, the three points of the object. The algorithm of Shi [17] combines color contrast and structural contrast. Liu [18] and other mathematical methods are adopted. Yao Zhaojian [19] proposes an algorithm for image saliency detection based on background prior and foreground information.

\subsection{Saliency Detection Model for Deep Learning Methods}

Unlike traditional visual saliency with handmade features, this is a fully data-driven CNN. Junting[19] provides an end to end convolution neural network algorithm for significant detection. Scholars Lu Huchuan and Cheng Mingming have used deep learning methods to do significant tests in recent years. FCN is widely used in saliency detection models, such as [20-24]. The network of deep learning requires a lot of data, and millions of levels are even tens of millions of , so migration learning plays an important role.

\section{Current Challenges and Future Prospects}

In addition to the traditional methods, the future saliency model algorithm can also use deep learning, and the challenges brought by deep learning also exist. For example, a lot of training data problems, the marking of training data is more complicated. But now the black box of deep learning has been opened. It enables people to understand deep learning and extract features further. The development of deep learning models, such as $\mathrm{rfcn}$ and SSD, also produces a new algorithm for saliency detection. The future algorithm for saliency detection is to be able to get better performance than before.

\section{Conclusions}

This paper mainly studies the model of image saliency detection. We make a summary of them, introduce various models and classify the models, including traditional methods and deep learning methods, the latest progress, the problems and challenges of saliency models, summarize and put forward future research directions. 


\section{References}

[1] Li J, Gao W. Visual Saliency Computation[J]. Lecture Notes in Computer Science, 2014, 8408(4):8-9.

[2] Achanta R, Hemami S, Estrada F, et al. Frequency-tuned salient region detection[C]// Computer Vision and Pattern Recognition, 2009. CVPR 2009. IEEE Conference on.IEEE, 2009:1597-1604.

[3]Movahedi V, Elder J H. Design and perceptual validation of performance measures for salient object segmentation[C]// Computer Vision and Pattern Recognition Workshops. IEEE, 2010:49-56.

[4]Cheng M M, Zhang G X, Mitra N J, et al. Global contrast based salient region detection[C]// Computer Vision and Pattern Recognition. IEEE, 2011:409-416.

[5]Fan D P, Liu J J, Gao S H, et al. Salient Objects in Clutter: Bringing Salient Object Detection to the Foreground[J]. arXiv preprint arXiv:1803.06091, 2018.

[6]Fan D P, Cheng M M, Liu Y, et al. Structure-Measure: A New Way to Evaluate Foreground Maps[C]// IEEE International Conference on Computer Vision. IEEE Computer Society, 2017:4558-4567.

[7]Qin Yao, based on the significance of cellular automata detection [C]. Dalian, Dalian University of Technology, 2007432-442.

[8]Qian Sheng, based on the saliency detection [C]. Journal of automation, airport and image segmentation are 2015, 41 (4): 711 - 724.

[9]Itti L, Koch C, Niebur E. A model of saliency-based visual attention for rapid scene analysis[M]. IEEE Computer Society, 1998.

[10]Liu T, Yuan Z, Sun J, et al. Learning to detect a salient object.[J]. IEEE Transactions on Pattern Analysis and Machine Intelligence, 2010, 33(2):353-367.

[11]Achanta R, Hemami S, Estrada F, et al. Frequency-tuned salient region detection[J]. 2009, 22(9-10):1597-1604.

[12]Judd T, Durand F, Torralba A. A Benchmark of Computational Models of Saliency to Predict Human Fixations[J]. 2012.

[13]Jiang H, Wang J, Yuan Z, et al. Salient Object Detection: A Discriminative Regional Feature Integration Approach[C]// IEEE Conference on Computer Vision and Pattern Recognition. IEEE Computer Society, 2013:2083-2090.

[14]Fu Y, Cheng J, Li Z, et al. Saliency Cuts: An automatic approach to object segmentation[C]// International Conference on Pattern Recognition. IEEE, 2008:1-4.

[15]Krahenbuhl P. Saliency filters: Contrast based filtering for salient region detection[C]// Computer Vision and Pattern Recognition. IEEE, 2012:733-740.

[16]Jiang P, Ling $\mathrm{H}, \mathrm{Yu}$ J, et al. Salient Region Detection by UFO: Uniqueness, Focusness and Objectness[C]// IEEE International Conference on Computer Vision. IEEE Computer Society, 2013:1976-1983. 
[17]Shi K, Wang K, Lu J, et al. PISA: Pixelwise Image Saliency by Aggregating Complementary Appearance Contrast Measures with Spatial Priors[C]// Computer Vision and Pattern Recognition. IEEE, 2013:2115-2122.

[18]

Liu R, Cao J, Lin Z, et al. Adaptive Partial Differential Equation Learning for Visual Saliency Detection[C]// Computer Vision and Pattern Recognition. IEEE, 2014:3866-3873.[19]Yao Zhaojian, Tan Taizhe. Combining the saliency detection [J]. background and foreground of the prior China Journal of image and graphics, 2017, 22 (10). [20]Pan J, Sayrol E, Giroinieto X, et al. Shallow and Deep Convolutional Networks for Saliency Prediction[J]. 2016:598-606.

[19]Yao Zhaojian, Tan Taizhe. Combining the saliency detection [J]. background and foreground of the prior China Journal of image and graphics, 2017, 22 (10). [20]Pan J, Sayrol E, Giroinieto X, et al. Shallow and Deep Convolutional Networks for Saliency Prediction[J]. 2016:598-606.

[21]G. Lee, Y.-W. Tai, and J. Kim. Deep saliency with encodedlow level distance map and high level features. In CVPR,pages 660-668, 2016. 1, 2, 6, 7.

[22]G. Li and Y. Yu. Visual saliency based on multiscale deep features. In CVPR, pages 5455-5463, 2015. 1, 2.

[23]N. Liu and J. Han. Dhsnet: Deep hierarchical saliency net-work for salient object detection. In CVPR, pages 678-686,2016. 1, 2, 5, 6, 7.

[24]L. Wang, H. Lu, X. Ruan, and M.-H. Yang. Deep networks for saliency detection via local estimation and global search. In CVPR, pages 3183-3192, 2015. 1, 2, 6, 7 R. Zhao, W. Ouyang, H. Li, and X. Wang. Saliency detection by multi-context deep learning. In CVPR, pages 1265-1274,2015. 1, 2, 6, 7 . 